\title{
The need for new regulations on randomized clinical trials in surgical treatments
}

\author{
Emmanuel Houdart
}

Received: 12 July 2010 / Accepted: 11 August 2010 / Published online: 25 August 2010

(C) Springer-Verlag 2010

\section{Dear Sir,}

Amongst the methodologies used to assess the value of a medical intervention, the randomized clinical trial provides the most reliable results. Its principle is well known and my concern and that of this letter are studies that compare the outcome of preventive surgical treatment to the natural history of a lesion. My intention is to show that without regulations mandating active participation of all planned study sites, randomized studies conducted in this setting are bound to fail.

It may appear unethical to apply randomization in this situation. Yet, both physicians and patients would be surprised to learn that many vascular abnormalities found fortuitously are preventively treated (and often very aggressively) even though the treatment's benefit has never been demonstrated. The possibility of a catastrophic treatment complication in a healthy patient whose untreated risk is not precisely known justifies the concept of such an evaluation. Various attempted randomized studies in this situation have failed because of lack of participation from surgical or interventional teams. I will illustrate the point with two recent neurological examples.

The first example involves unruptured cerebral aneurysms that are more and more frequently discovered as noninvasive medical imaging methods (CT scan and MRI) develop. In most cases, their treatment by coil embolization (intravascular surgery) is possible. Yet, two major questions remain unanswered: (1) is treatment-related risk in a nonsymptomatic patient acceptable when the rupture risk is not

\section{E. Houdart $(\triangle)$}

Department of Neuroradiology, Lariboisière Hospital,

2 rue Ambroise Paré,

75475 Paris Cedex 10, France

e-mail: emmanuel.houdart@lrb.aphp.fr well known?, and (2) is coil embolization protection long lasting? The TEAM study [1] sponsored by the Canadian Institutes of Health Research attempted to answer these questions, but failed because of the lack of participation from the interventional neuroradiology teams.

A similar failure seems to be coming with the ARUBA study [2] that attempts to answer the same questions on the treatment of unruptured brain arteriovenous malformations. This study is organized and sponsored by the National Institutes of Health and was approved by 60 Ethics committees and Institutional Review Boards in January 2008, when inclusions started. In June 2010, the study is threatened by the very low accrual rate.

Many neuroradiological teams have refused upfront to participate in both studies because of various arguments, none of which hold up to elementary analysis. "The study is not ethical". Health Authorities should therefore be informed that the Government (Canadian for TEAM and USA for ARUBA) uses public funds to finance unethical studies, and that the Ethics Committees that approved them are ethically incompetent. "The study is not valid as the results of good centers will be confounded by those of mediocre centers". In real life, all patients are not all treated in the top centers (defined on which criteria?), and the results of treatment must take into account all qualified centers. Besides, in both studies discussed here, the centers were carefully selected and were, for most of them, specialized university centers. "The study does not take into account certain individual factors that may increase the lesion's natural risk". Wrong, these factors were pre-defined in the study protocols prior to starting recruitment.

This last argument shines some light on the preconception that many interventionists or surgeons have about the conclusions that may be drawn from these studies. The answer to the preventive intervention benefit question is 
thought to be binary, i.e., a "global" yes or no. However, if the randomization has balanced the subgroups between the two study arms, and if there is a difference in the natural history of the lesion in one or more of these subgroups, this difference should show up in the study results, provided that this analysis has been planned.

The real reasons for this refusal to participate (or what may be worse, for the pretense of participation that most centers have been displaying) are of a different nature. It is easy to understand that such studies by definition lead to a decrease in the interventional activity (a minimum of 50\% of patients do not undergo the procedure). This decreased activity has several consequences, none of which provides an incentive towards active participation.

The first consequence is economic: in some cases, loss of honorarium for the operator, in others, loss of revenue to the institution itself. How can the cost of buying or upgrading expensive equipment (angiography or radiotherapy apparatus) be justified to one's institution if that equipment is under-utilized? These costs are high and are unlikely to be offset by the study Sponsor. The second consequence may well be the operator's fear that he or she may have to reconsider the need for an intervention which has taken years to master and through which the therapist's practice has acquired recruitment and recognition. Lastly, the risk of losing the specialty accreditation that the Department obtains from its Society by performing a yearly critical number of procedures. These non-monetary consequences may not be offset by the publication of a multiauthored paper presented in the distant future, after the large number of patients have been followed up for up to 10 years.

How then can we be surprised at the lack of participation in such a study if participation is optional? As good intention will never triumph over human self-interest, this is where the reason for failures to enroll must be sought.

For the medical community, the choices are simple. We should either stop setting up such studies or change the rules of participation. If the choice is to stop, this has to be signified clearly, to the medical community, the patients, and the health authorities, so that time and money are not spent in setting up future studies that are bound to fail.

If the choice is to pursue this line of clinical research, then, new rules must be set-up and agreed upon. This conflict between the individual and general interest is not new, and has been written of by Emmanuel Kant [3] who also gave the fundamental principles needed to resolve it. "A multitude of reasonable beings desire for their preservation universal laws, though everyone among them has a secret inclination to except himself from the observation of them. A constitution must therefore be given them, that so confines their individual passions, one by means of the other, that, in their public conduct, their effect becomes as inconsiderable as if they had not these hostile dispositions".

Isn't "the multitude of reasonable beings" the medical community, which all have a natural tendency to secretly exclude themselves from the law that is necessary to the conservation of their specialty? How can we justify the cost of an intervention to Health Authorities if a benefit to the patient is not established?

When the benefit of an intervention is clearly questioned by the medical community, every surgical center should be required to submit the protocol to its Ethics Committee. If the majority of these Ethics Committees approves the protocol, then this decision would render participation of all the relevant sites mandatory.

Enforcement would be simple: reimbursement of the procedure would be linked to the patient's inclusion into the study.

I know how traumatic enforced participation may be, both for the patient and for the physician, but the question has been raised by our individual practices, and it needs to be answered. That question, simply put, is "what is the constitution needed for our preservation and that of our patients?"

Conflict of interest statement I declare that I have no conflict of interest.

\section{References}

1. Trial on endovascular aneurysm management, NCT00537134, http://clinicaltrials.gov/ct2/show/NCT00537134

2. A randomized trial of unruptured brain AVMs, NCT00389181, http://clinicaltrials.gov/ct2/show/NCT00389181)

3. Emmanuel Kant (1932) Perpetual peace. Columbia University press, New York 